Enfoques. Revista de Investigación en Ciencias de la Administración http://doi.org/10.33996/revistaenfoques.v3i12.70 No. 12 | Volumen 3 | Octubre - Diciembre 2019 http://revistaenfoques.org

\title{
ESTRATEGIA DE ENDOMARKETING PARA FIDELIZAR A LOS CLIENTES INTERNOS DE UNA EMPRESA DE PIZZA
}

\author{
ENDOMARKETING STRATEGY TO BUILD LOYALTY TO \\ INTERNAL CUSTOMERS OF A PIZZA COMPANY
}

\author{
Roxana Marlene Apaza Mamani
}

\section{Resumen}

El articulo abarca el objetivo de coadyuvar con la fidelización de los clientes internos de la empresa pizzería "El Pizzarrón" en la ciudad de Potosí, tomando como base los principios fundamentales, los requerimientos que tiene la empresa y asimismo sus clientes internos, sobre el tema de fidelización, ya que entre ambas partes existe un vínculo laboral. Para ello se desarrollaron lo estudios necesarios de las distintas teorías existentes del endomarketing, apoyado en los métodos de nivel teórico y empírico, los que brindan una descripción clara de la fidelización del cliente interno, basado en la metodología científica para el presente trabajo de investigación. Se ven reflejando los resultados obtenidos que arrojan información que corrobora la existencia del requerimiento por parte de la empresa, en cuanto al tema de fidelización de los clientes internos. La valoración de resultados aporta información valiosa, la cual permite que se proponga una estrategia de endomarketing, fundamentadas en fases, actividades y acciones para su implementación, misma que permitió la fidelización de los clientes internos de la empresa pizzería "El Pizzarrón" de la ciudad de Potosí.

Palabras clave: Fidelización de clientes; estrategias de endomarketing; requerimiento empresarial

\begin{abstract}
The article covers the objective of helping with the loyalty of the internal customers of the pizzeria "El Pizzarrón" in the city of Potosí, based on the fundamental principles, the requirements that the company has and also its internal customers, on the subject of loyalty, since between both parties there is a labor link. For this, the necessary studies of the different existing theories of endomarketing were developed, supported by theoretical and empirical methods, which provide a clear description of the loyalty of the internal client, based on the scientific methodology for the present research work. They are reflecting the results obtained that provide information that corroborates the existence of the requirement on the part of the company, regarding the subject of loyalty of internal customers. The valuation of results provides valuable information, which allows an endomarketing strategy to be proposed, based on phases, activities and actions for its implementation, which allowed the loyalty of internal customers of the pizzeria "El Pizzarrón" in the city from Potosí.
\end{abstract}

Key words: Customer loyalty; endomarketing strategies; business requirement
Roxana Marlene Apaza Mamani roma.apaza@gmail.com Universidad Privada Domingo Savio, Bolivia

Ingeniero Mecánico, egresado de la Universidad del Zulia. Magister Scientiarum en Gerencia de Operaciones. Universidad del Zulia. 


\section{INTRODUCCIÓN}

En la actualidad el mantenimiento de las empresas en un mercado competitivo involucra la constante renovación de políticas y criterios que vayan en función de involucrar al empleado, o también considerado cliente interno como una parte esencial de la organización de la empresa, por ende, el estado en el que se encuentre el cliente interno en la empresa determinara de gran forma los resultados de las operaciones y por lo tanto condicionara el nuevo funcionamiento de la misma.

Uno de los inconvenientes con lo que se atraviesa en las empresas es el abandono de los trabajadores a la fuente laboral cuando se comienzan a familiarizar con los procedimientos para los que fueron contratados, este hecho involucra la necesidad de contratación de nuevo personal y la capacitación del mismo convirtiéndose este hecho en una carga para la empresa.

Existen varios factores que pueden estar implícitos en la decisión que puede tomar una persona para abandonar un trabajo, estos factores van desde lo personal, familiar, económico, social y de crecimiento; dichos factores provocan en la persona una situación de confrontación entre el cliente interno y su realidad circundante.

En ese sentido, el endomarketing ofrece una perspectiva de solución a dichas circunstancias, ya que provee de la posibilidad de hacer marketing al interior de la empresa, donde a través de los criterios de acción promueve en los clientes internos una perspectiva de pertenencia a la empresa, proveyendo de identidad hacia la misma y garantizando el establecimiento de una relación perdurable entre el cliente interno y la empresa.
El principal beneficio del endomarketing, es que los resultados rompen límites de su propia área, es decir, logra efecto de coordinación entre las diferentes áreas de la organización, lo cual permite el desarrollo y la correcta implementación de los planes y estrategias que así se hayan definido, generando y cubriendo expectativa $y$ satisfacción primero dentro y luego fuera de la empresa.

En la ciudad de Potosí, existen una gran cantidad de empresas de producción de alimentos, enmarcadas en la oferta de comida rápida, una de ellas es la Pizzería "El Pizarrón" empresa de origen local, establecida hace aproximadamente dos décadas y que ha presentado un considerable crecimiento habiendo llegado a constituir una nueva sucursal, teniendo dos locales de venta en la ciudad. Dicha empresa presenta problemas por la deserción continua de los empleados (cliente interno) y precisa de una estrategia para mantener un parámetro de mantención de los mismos por periodos de tiempo más amplios.

Se ha establecido también que uno de los indicadores de alarma es la existencia de ausencia, debido a tiempos en los que por la deserción de empleados no se ha podido cubrir las vacantes existentes durante un periodo considerable de tiempo, hecho que involucra el aumento de asignaciones para los empleados activos que deben cubrir el trabajo generado por la ausencia laboral.

Es las razones antes mencionadas que se plantearon un conjunto de interrogantes relacionadas con ¿Cómo lograr la fidelización del cliente interno de la pizzería "El Pizarrón" de la ciudad de Potosí? Partiendo teniendo como objeto de estudio la Fidelización. Además el campo de acción se fundamentó en la fidelización del cliente interno de la pizzería “El Pizzarrón” en la ciudad de Potosí. 
Por lo tanto, para el desarrollo de este estudio se elabora una estrategia de endomarketing para la fidelización del cliente interno de la pizzería "El Pizzarrón" de la ciudad de Potosí. Con la finalidad de orientar la resolución del problema científico y dar cumplimiento al objetivo

En correspondencia a las preguntas científicas se plantea las siguientes tareas científicas:

- Sistematización de los fundamentos teóricos de la fidelización y la estrategia de endomarketing.

- Caracterización del estado actual de la fidelización del cliente interno de la pizzería "El Pizzarrón" en la ciudad de Potosí.

- Elaboración de una estrategia de endomarketing para la fidelización del cliente interno de la pizzería "El Pizzarrón" en la ciudad de Potosí.

La importancia de este estudio con relación a la aplicación de la teoría como dispositivo y estrategia al endomarketing como parte del marketing, es importante, ya que la teoría al respecto, advierte la importancia de satisfacer al cliente/mercado, pues bien el endomarketing tiene el mismo efecto, pero que la misma empieza al interior de la empresa, lo cual sugiere justo y coherente, el aporte a los propósitos de toda actividad comercial se refleja en la contribución de líneas de acción e instrumentos propios para la plena satisfacción y conformidad del cliente interno, siendo particularmente de especial importancia para atender las necesidades que se ha podido observar en la pizzería "El Pizzarrón", contribuyendo a su vez con la teoría pertinente y acorde a la empresa respecto a fidelización y endomarketing, puesto que actualmente no existe una investigación de esta magnitud con relación a ello para la empresa.

El endomarketing orientado a conocer la manera más apropiada para su aplicación y la fidelización de los clientes internos de la pizzería "El Pizzarrón", lo cual significa un aporte cualitativo y estratégico para esta empresa, beneficiando en sustentar la fidelización de la forma más adecuada a los clientes internos. Brindando de esta manera soluciones, como opciones a ser aplicadas en forma inmediata, siendo ello consistente para utilización como alternativa debidamente sustentada y apoyada en la presente investigación.

La investigación contribuye a revelar las diversas características que tienen los clientes internos de la empresa, al momento de decidir ser capacitados, relacionarse entre ellos y con su inmediato superior en el ambiente de trabajo. Además de ello ira a fortalecer y coadyuvar a la empresa y brindando calidad de vida a las personas mediante trato al cliente interno, repercutiendo asimismo con los clientes externos que se vayan a relacionar los clientes internos por medio del trato que los mismos vayan a otorgar, o más conocido como la "atención al cliente", que es tan esperado por los clientes externos.

\section{MÉTODO}

Se sustentó bajo el enfoque mixto. Es decir, el método cualitativo, mismo que consiste en la identificación, valoración y caracterización de los atributos y sus condicionantes de la información dirigida y recabada apropiada y propia del tema, es decir, en este caso de la fidelización. El tipo de investigación que se asume, es el descriptivo, ya que se realizó un análisis de la situación problemática, identificándose los hechos a ser estudiados con relación a la 
fidelización de la pizzería "El Pizarrón" y a partir de ello, visualizar de manera substanciosa posible solución a la misma.

\section{RESULTADOS Y DISCUSIÓN}

\section{Fase diagnóstica}

Después de realizada la entrevista la información que se considera la destacable, se menciona para entender cierto grado de proporción acerca de lo que era la situación con relación a la fidelidad por parte de los clientes internos, dicha información se aprecia a continuación:

En relación al perfil del cliente interno señaló que:

- Se apreció que un considerable grupo de clientes internos se encuentra en proceso de formación académica, es decir, se encuentra estudiando.

- Los contratos realizados por la empresa han sido verbales, por lo que los clientes internos no han atribuido el nivel de seriedad adecuado a la empresa.

- Una gran parte de los clientes internos no tiene una relación de exclusividad con la empresa, sino que cuentan al mismo tiempo con otra actividad.

- La mayoría de los clientes internos ha tenido reclamos tener que cubrir funciones de los puestos vacantes en la empresa.

En cuanto al comportamiento del cliente indica lo siguiente:

- Una gran parte de los empleados no tienen mucho tiempo en la empresa.

- Se considera que los empleados cumplen con su trabajo medianamente, y que solamente un par se preocupa por el bienestar de la empresa.
- Los empleados no se relacionan con la misión ni la visión de la empresa.

- La mayoría no brinda seguridad e cuanto al tiempo que permanecerá en la empresa.

En relación a la relación empresa cliente:

- Considera que los empleados se encuentran satisfechos con su trabajo, puesto que muy pocas veces se presentan quejas o inconveniencias.

- El gerente propietario señala que siempre se han esforzado por proveer de un buen trato a los empleados, tratando de que se sientan cómodos en el trabajo.

- Indica que los incentivos que se les da implican las temporadas del año, especialmente en fin de año se les entrega un canastón navideño y a los empleados que presentan la antigüedad se les otorga un aguinaldo.

- Se indicó que siempre se les concede un tiempo para aprender la forma adecuada de realizar su trabajo.

Después de un análisis detallado de los componentes del fenómeno en la empresa, se determinó que:

La empresa está al tanto de cómo el personal se retira de la misma, por lo que en el proceso de tener una relación más estrecha, viendo desde el punto de vista de la teoría del endomarketing con sus clientes internos no es el más adecuado, ya que se debe establecer una relación firme entre ambas partes desde el principio. La efectividad de la misión y visión de la empresa en la misma no es buena, puesto que los clientes internos en un alto porcentaje no conocen la razón de ser de la empresa y tampoco a dónde quiere llegar y 
por consecuencia no existe una relación intrínseca para con la empresa por parte de los clientes internos.

También se pudo identificar que la empresa se apoya en el pago remunerado como principal incentivo para con sus clientes internos, a pesar de que se brindan otro tipo de incentivos que no son considerados como algo de la empresa, ya que el aguinaldo y canastón, que están descritos como parte de los incentivos de la empresa se realizan en distintas instituciones, solo las convivencias recreativas pudiese considerarse como algo propio de la empresa que busca mantener incentivados a los clientes internos.

Los clientes internos cuentan con otras ocupaciones como estudios y otro trabajo, de lo cual la empresa tiene conocimiento, por lo que su fidelidad se encuentra afectada, debido a estas razones no existe una permanencia duradera en la empresa y las faltas laborales por parte de los clientes internos, hace que ello no sea beneficioso para la empresa.

No existen capacitaciones como manifiestan el empleador y los clientes internos, sin embargo, si se cuenta con una inducción al inicio del trabajo a realizar en la empresa, estas solamente son impartidas por los mismos clientes internos 0 el empleador de la Pizzería.

Uno de las situaciones que se presenta en la empresa es el retiro o migración que realizan los clientes internos por diversos motivos, uno de estos motivos es la posibilidad de ir a trabajar a una empresa semejante a la pizzería "El Pizarrón", propiamente la competencia, siendo ello, algo desfavorable para la empresa después de haber invertido diversos recursos en los clientes internos que se van.

\section{Fase de diseño y elaboración de una estrategia de endomarketing para la fidelización del cliente interno de la pizzería "el Pizzarrón" en la ciudad de potosí}

\section{Introducción}

La elaboración de una estrategia de endomarketing debe tomar en cuenta la comunión entre esta básica herramienta y la fidelización del cliente interno, de tal forma se genere y se desarrolle escenarios de empoderamiento, compromiso, los necesarios para mejorar niveles de rendimiento expectables par la empresa como plena satisfacción para los clientes internos.

Cuando se tiene un equipo de cliente interno que termine definiendo prosperidad con este tipo de desafíos, en el marco de lo expresado por Gómez (2014), donde el endomarketing: "es hacer marketing al interior de la empresa", hecho que representa cierta dificultad, debido a que cuando un cliente interno llega a ser parte de la empresa este accede a todos los beneficios que le brinda la empresa, automáticamente se llega a tener una relación en la que tanto la empresa y como el cliente interno buscan satisfacer sus necesidades organizacionales como laborales respetivamente. Entonces, queda preguntar, ¿Dónde está la desventaja de esta relación? Y la respuesta es clara....en la existencia y grado de la fidelidad.

No es suficiente que el cliente interno simplemente se relacione con la empresa en el sentido estricto del cumplimiento de sus obligaciones, sino que establecer también de forma personal establezca un compromiso de fidelidad hacia la empresa, dicho compromiso de fidelidad apunta a una relación duradera y exclusiva. 
Entonces, se debe tomar en cuenta el plano de fidelización del cliente interno, como sostiene Nayar (2011):

... cuando una compañía pone a los clientes internos en primer lugar, el cliente pasa realmente a ocupar el primer lugar en última instancia y a obtener el máximo beneficio, pero de un modo bastante más transformador que a través de los clásicos programas de «atención al cliente» $y$ otros parecidos. (s/p).

\section{Justificación}

Tras haber realizado un diagnóstico del estado actual de la fidelización de los clientes internos de la pizzería "El Pizarrón" en la ciudad de Potosí, se ha llegado a definir la columna vertebral del grado de relacionamiento y la forma como debe ser encarada en la empresa, debiendo ser éstas adecuadas y duraderas, para este efecto se hace necesario estratificar indicadores que se constituyen en información base, como resultado de los procesos de análisis concretizado en este mismo documento:

- Aspecto considerado en riesgo o en desventaja:

- No existe proyección de relación a largo plazo por parte del cliente interno, siendo vulnerable de tomar otras opciones si es que acaso se presentase.

- Desconocen el marco de intenciones, así como las estrategias, metas y grado de contribución que ellos constituyen para con la empresa.
- Aspecto considerado en conveniencia o en ventaja:

- Plena satisfacción en cuanto al clima organizacional al interior de la empresa, el trato, cortesía, consideración y grados de compañerismo se evalúan aceptables.

- Existencia de conformidad, a pesar que de que se tiene la obligación por norma con relación a los beneficios que recibe, pero también con la remuneración que reciben.

Estos términos conclusivos, favorables y desfavorables, se constituyen en razones suficientes para emplazar una estrategia coherente y apropiada que hagan de los riesgos una reingeniería de conductaprocedimiento y de las ventajas una base sólida para siempre mejorar y perfeccionar lo que sea necesario para conquistar $y$ mantener fidelización con el cliente interno

\section{Diseño de la propuesta}

La propuesta implica establecer los lineamientos para el diseño de una estrategia que lleve a los clientes internos de la empresa pizzería "El Pizarrón" a establecer mejores relaciones internas en la empresa, y que estas relaciones estén sujetas a la fidelidad, por tal motivo se toma en cuenta los aspectos del endomarketing y la fidelización del cliente interno

- Planteamiento de objetivos

- Detallar las necesidades del cliente interno

- Estrategia de endomarketing 


\section{Objetivo General}

Diseñar una estrategia de endomarketing para incrementar el nivel de fidelización del cliente interno en la empresa pizzería "El Pizarrón" en la ciudad de Potosí.

\section{Estrategia de endomarketing}

De acuerdo a la modelo de Regalado, Allpacca, Baca y Gerónimo, de manera detallada amplían el proceso de implementación de la estrategia de endomarketing, el cual está basado en las siguientes fases:

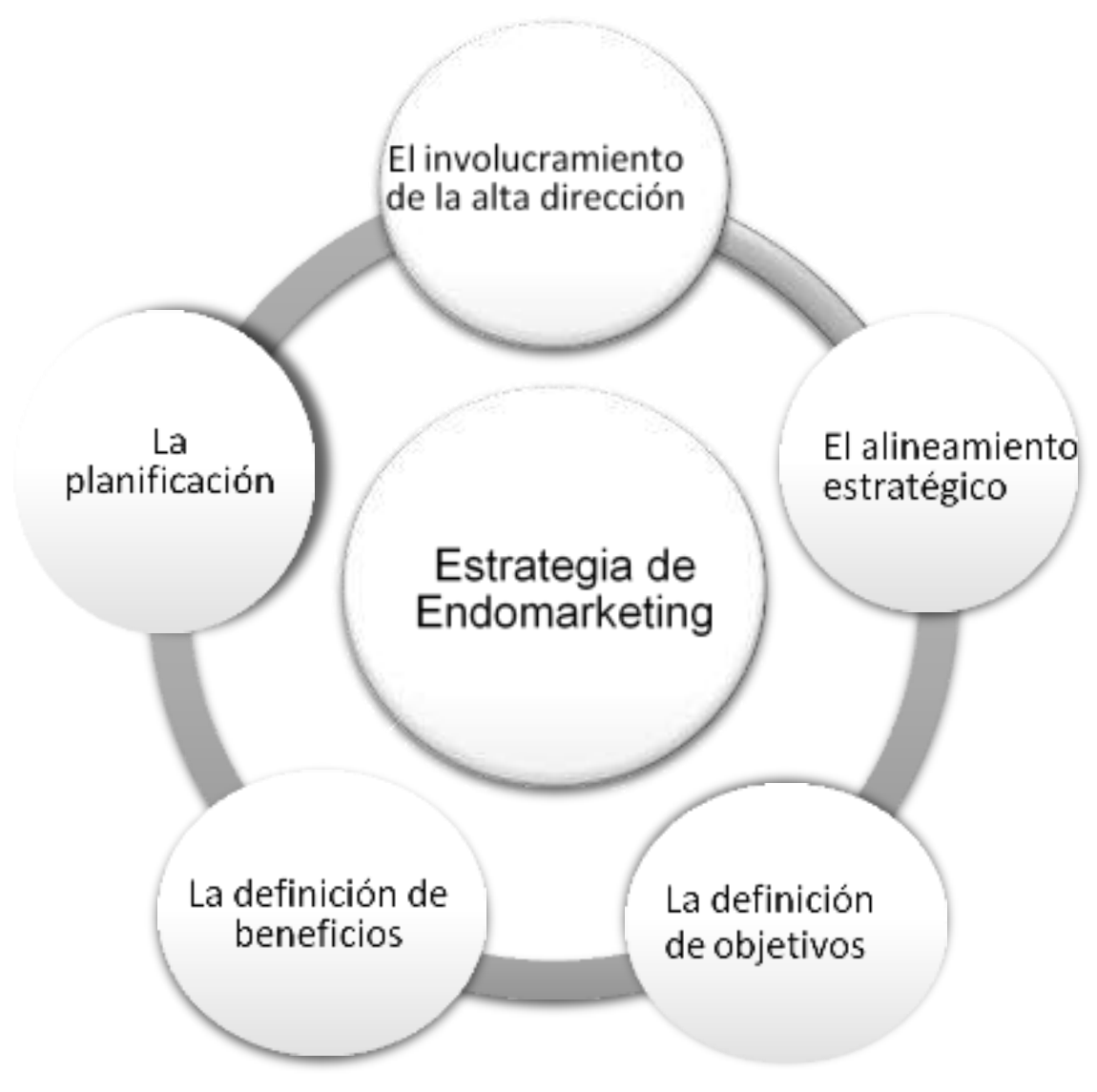

Figura 1. Fases de implantación de la estrategia endomarketing. 
Desarrollo de la estrategia de endomarketing

A continuación se muestran las fases para la implementación de la estrategia en la empresa.

\section{El involucramiento de la alta dirección}

Se desarrolló por medio de las siguientes actividades:

- Permiso "Político".- Reuniones de validación y socialización de los planes a implementarse en la empresa.
- Compromiso organizacional.- Equipo gerencial a cargo de la implementación de la estrategia.

- Involucramiento del empleado.Relacionamiento con la gerencia para efectuar y realizar los distintos planes y metas.

- Liderazgo de recursos humanos y marketing.- Vinculación de toda la organización en el conocimiento de las actividades y planes, para su adecuada ejecución.

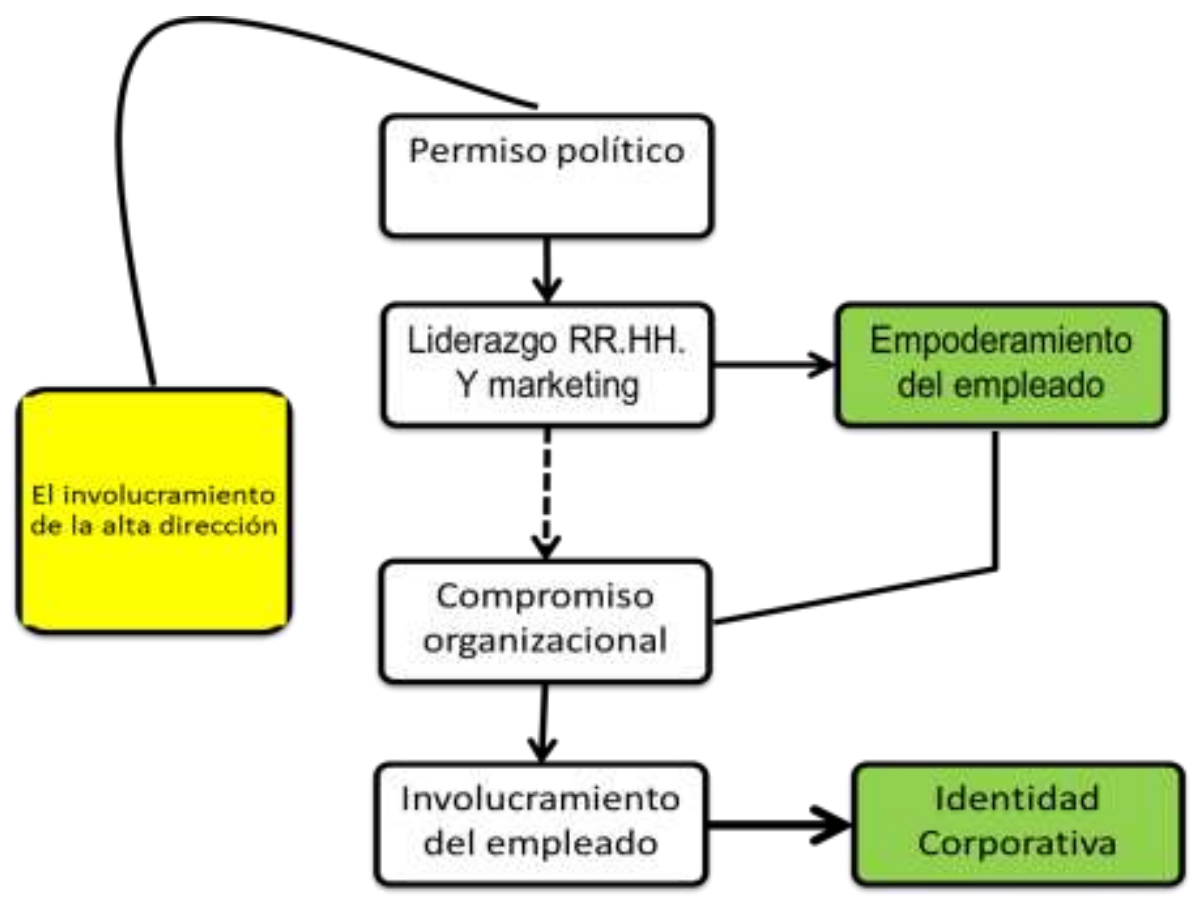

Figura 2. Dinámica de involucramiento en alta dirección. (Elaboración propia en base a Regalado, Allpacca, Baca y Gerónimo, 2011) 
Para tal efecto se sigue la siguiente dinámica:

\section{El alineamiento estratégico}

En este punto se orienta a lograr un impacto en la rentabilidad de la empresa, por lo que se realizaron las siguientes actividades:

- Alineación con la estrategia empresarial.Conocimiento de la identidad de la empresa por medio de la misión y visión.

- Identificar al cliente interno.- Realizar una base de datos con la identificación personal de cada cliente interno.
- Cultura de la equidad.- Vinculación de la empresa mediante la misión y visión por parte de todos los que conforman la empresa.

- Involucrar a todas las áreas.- Fijar metas y establecer planes para la parte gerencial y operativa de la empresa.

Para tal efecto se ha diseñado una dinámica de interacción en la que interviene el proceso que se realiza para lograr el fin de toda la fase, que es el empoderamiento del cliente interno. Los pasos se detallan en la siguiente figura:

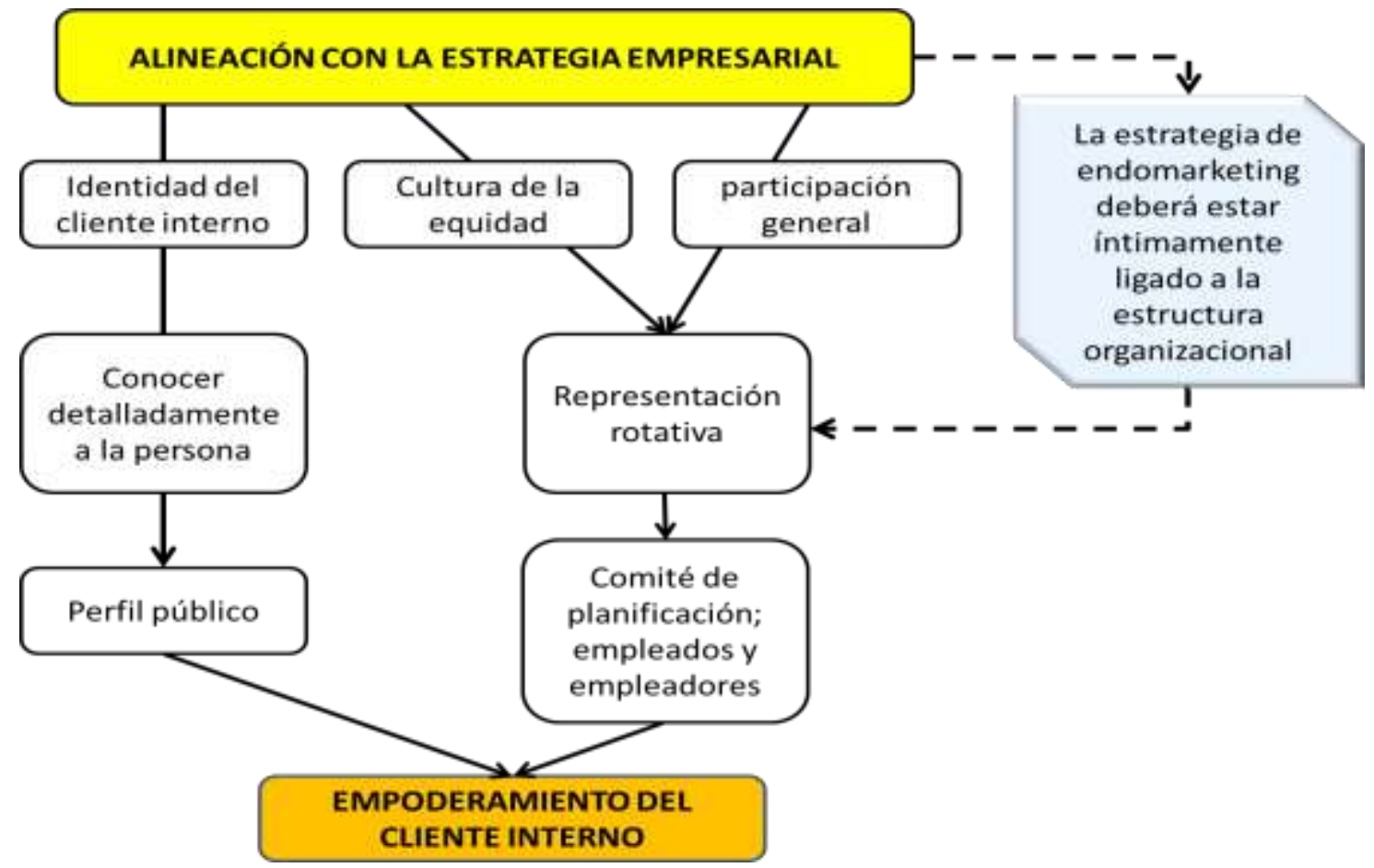

Figura 3. Dinámica de alineamiento estratégico.

(Elaboración propia en base a Regalado, Allpacca, Baca y Gerónimo, 2011) 


\section{La definición de objetivos}

Deben estar en línea con los objetivos de la empresa y expresar un beneficio genérico para todos los miembros de la organización.

- Identificar objetivos.- Los objetivos que persiguen la empresa tanto de funcionalidad, crecimiento y económicos.

- Definir indicadores.- Por medio de la fijación de metas y planes para el cliente interno.
- Empleado fidelizado.- Verificación y evaluación del desarrollo de las actividades dirigidas al cliente interno.

- Señala que los elementos con los cuales está conformado, deben ser claros para el cliente interno, quien al conocer los objetivos que se desea alcanzar la empresa, como el crecimiento de la empresa y el crecimiento de ellos mismos como cliente interno.
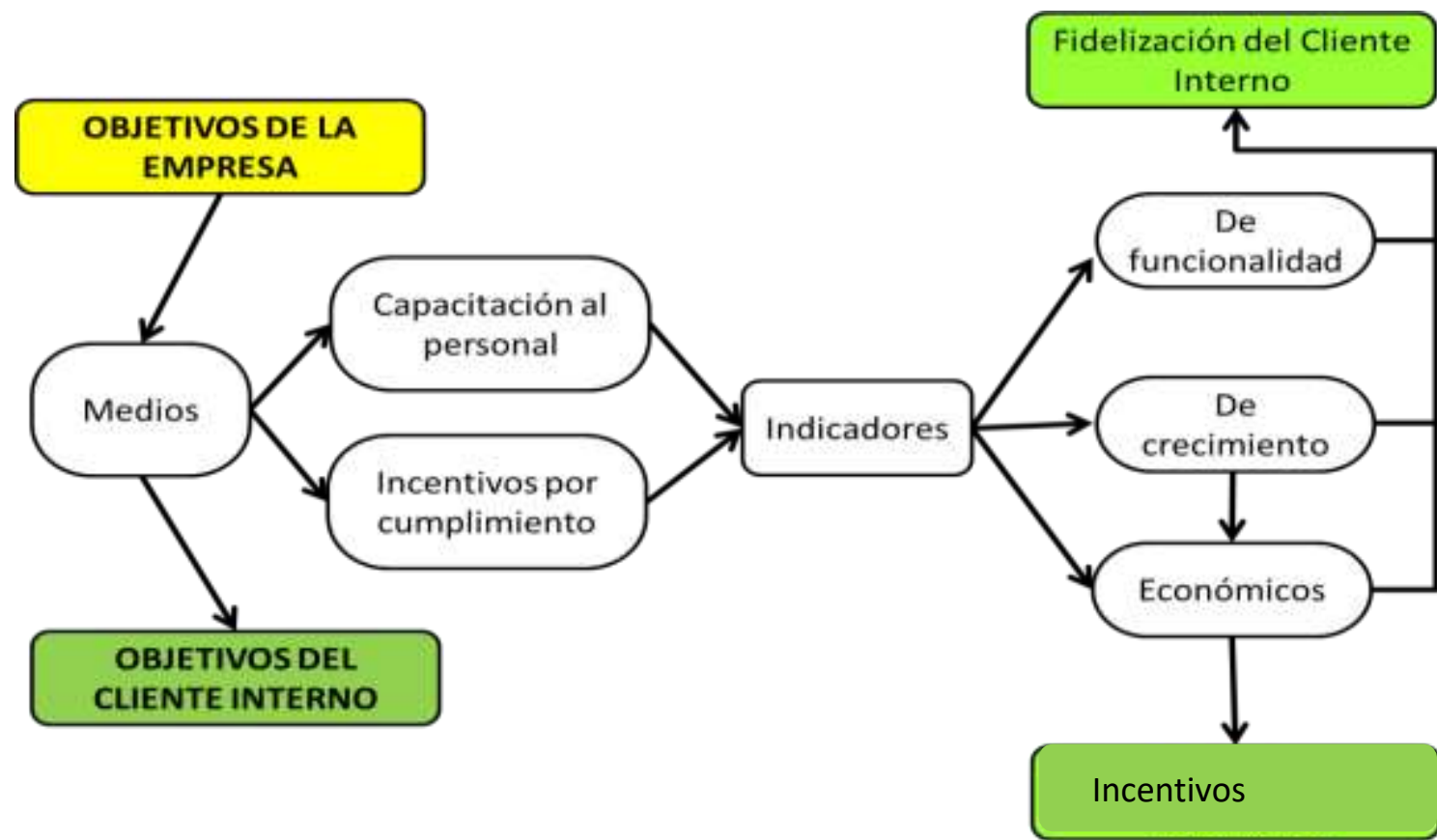

Figura 4. Dinámica para la definición de objetivos.

(Elaboración propia en base a Regalado, Allpacca, Baca y Gerónimo, 2011)

\section{La definición de beneficios}

Identificación de beneficios. Los que se procuraran son de crecimiento, económicos y de desarrollo, tanto para la empresa como para los clientes internos.

Beneficio mutuo. De tipo económico y de desarrollo, por motivo de que es la relación primordial que tiene la empresa.

Capacitación. En los aspectos concernientes al trabajo que se realiza en la empresa como atención al cliente, manejo de alimentos, etc.

Integración.

Realizar

la

retroalimentación necesaria en las áreas de la empresa. 
Gestión humana. Tener un representante de los clientes internos, que manifieste los requerimientos y necesidades concernientes al trabajo desarrollado en la empresa.

Debe contener elementos objetivos y subjetivos para el cliente interno, quien debe encontrar sentido a los beneficios que obtiene de la empresa, como sueldo, incentivos, programas motivacionales y de desarrollo personal.

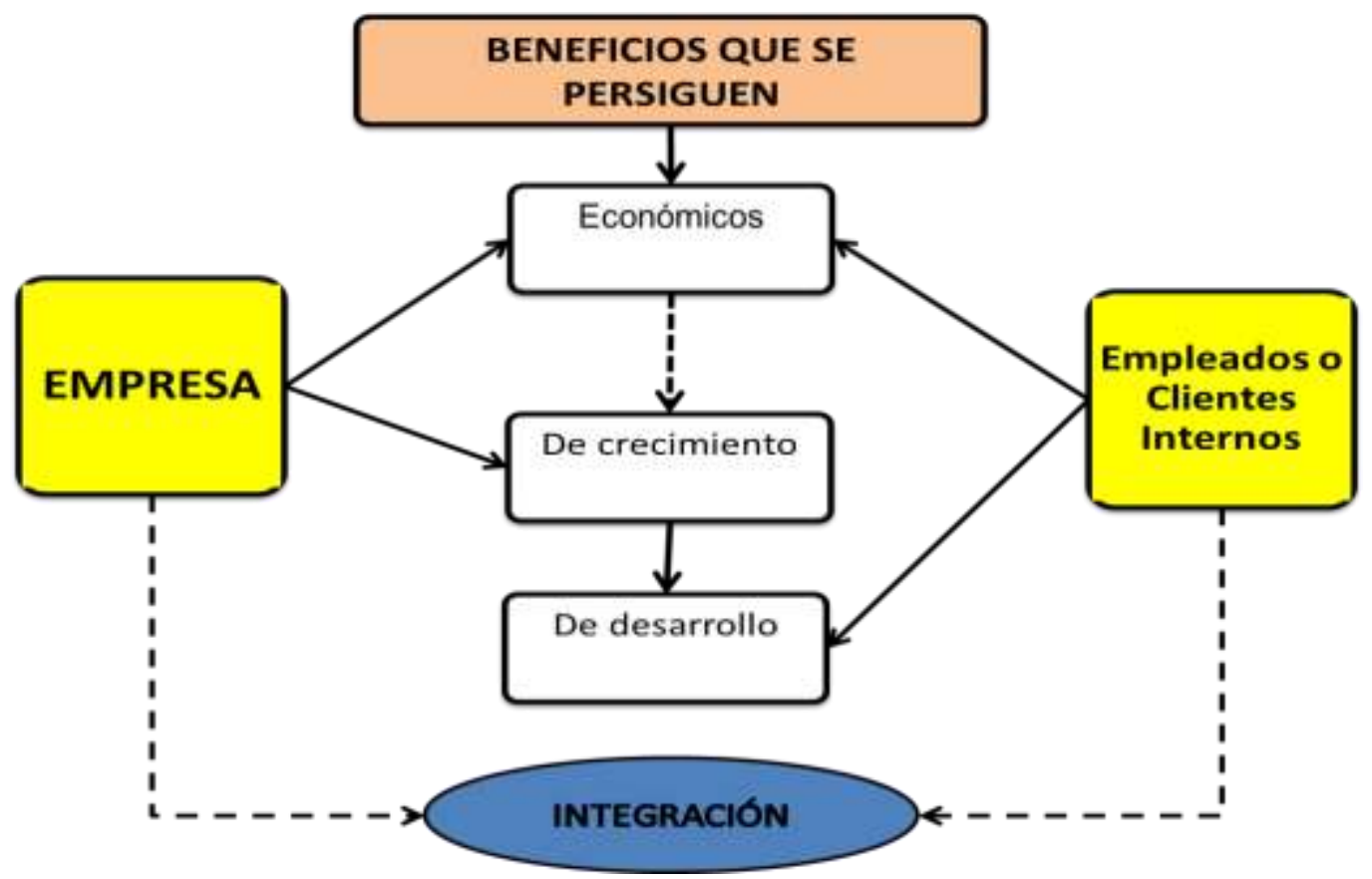

Figura 5. Dinámica de definición de beneficios.

(Elaboración propia en base a Regalado, Allpacca, Baca y Gerónimo, 2011)

\section{La planificación}

Se refiere a la definición de las etapas de la implementación de la estrategia de endomarketing propuesta, teniendo en cuenta que el proceso debe ir de arriba hacia abajo, y también de abajo hacia arriba para garantizar la cobertura en la empresa. Esto debe reflejarse en un calendario para que los empleadores como los clientes internos lo puedan cumplir.
- Comunicación efectiva. Una comunicación efectiva es vital, misma que debe efectuarse de manera constante en la empresa.

- Segmentación.- para lograr tener grupos funcionales y efectivos de clientes internos dentro la empresa. 
- Gestión de resistencias y grupos de influencia.- Generar representantes de los cliente internos, que coadyuven en la aplicación de la estrategia y se constituyan un referente para los demás clientes internos, siendo una asignación rotativa.

- Flexibilidad y adaptabilidad.- La flexibilidad y la adaptabilidad para con los clientes internos, son condiciones necesarias para que la estrategia sea aplicable a la empresa.
- Evaluación.- Por parte de los clientes internos y gerencia, con el propósito de verificar y adaptar la estrategia de forma evolutiva.

Cada una de estas etapas mencionada se enmarcan dentro del desarrollo de la estrategia de endomarketing, por la naturaleza de la misma se tiene que efectuar de manera constante, para su correcto funcionamiento. Por ese motivo en la siguiente figura se detalla cada una de las etapas de forma dinámica:

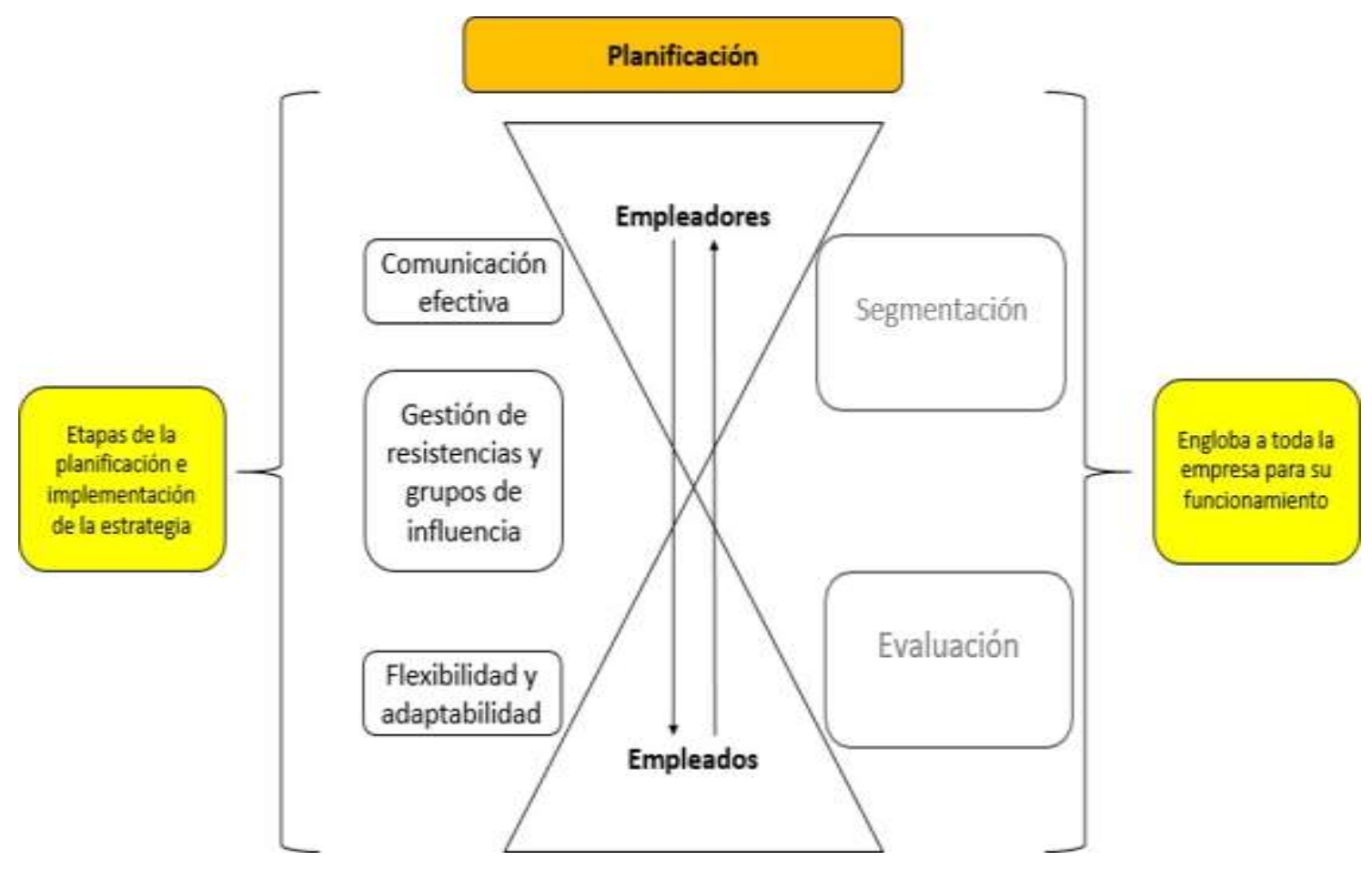

Figura 6. Dinámica de la planificación.

(Elaboración propia en base a Regalado, Allpacca, Baca y Gerónimo, 2011)

Actividades de la estrategia de endomarketing para fidelizar a los clientes internos

Dentro de la presente estrategia que se plantea a ser realizada, se involucran elementos como ser; materiales, tiempo y frecuencia con la que se efectuaran. Con el propósito de conocer las mismas de cada etapa de la estrategia a continuación se las menciona con mayor detalle. 
Estas actividades deben estar ligadas a los valores de la empresa, y dirigidas a satisfacer las necesidades de filiación y desarrollo que tiene los trabajadores, motivándolos a desarrollarse laboralmente a través de actividades de mutua colaboración con los empleadores.

Tabla 1. El involucramiento en la alta dirección

\begin{tabular}{|c|c|c|c|c|c|}
\hline Actividad & Desarrollo de la actividad & Emplazamiento & Materiales & Costo en Bs.- & Frecuencia \\
\hline $\begin{array}{l}\text { Permiso } \\
\text { "Político" }\end{array}$ & $\begin{array}{l}\text { Asignación de roles a } \\
\text { los clientes internos } \\
\text { para los equipos de } \\
\text { planificación }\end{array}$ & Empresa & Libro de actas & 20 & Única \\
\hline $\begin{array}{l}\text { Compromiso } \\
\text { organizacional. }\end{array}$ & $\begin{array}{l}\text { Conformación de un } \\
\text { equipo gerencial para la } \\
\text { planificación. }\end{array}$ & Empresa & Libro de actas & 20 & Única \\
\hline $\begin{array}{l}\text { Involucramiento } \\
\text { del empleado }\end{array}$ & $\begin{array}{l}\text { Conformación de un } \\
\text { equipo de planificación } \\
\text { de los clientes } \\
\text { internos con roles }\end{array}$ & Empresa & --- & 0 & Mensual \\
\hline $\begin{array}{l}\text { Liderazgo de } \\
\text { recursos } \\
\text { humanos y } \\
\text { marketing }\end{array}$ & $\begin{array}{l}\text { Rotación de los roles } \\
\text { del equipo de clientes } \\
\text { internos }\end{array}$ & Empresa & --- & 0 & Mensual \\
\hline
\end{tabular}

Nota: Elaboración propia en base a Regalado, Allpacca, Baca y Gerónimo, (2011)

\section{Acciones}

Permiso "Político". En el cual se involucra a la organización en su conjunto; es por ello, que se especifica los siguientes procedimientos a realizar:

$\checkmark$ Se realizará reuniones mensuales, siendo esta en la primera semana del mes, con el objeto de realizar retroalimentación de las acciones efectuadas, las cuales se desea implementar y mejorar los planes establecidos previamente, mismos que serán registrados en el libro de actas designado para ello.

Compromiso organizacional. La Gerencia se involucrará en el desarrollo de las siguientes acciones:

Se conformara un equipo gerencial, realizándose esto de forma semestral, siendo registrada esta función en un libro de actas, para el desarrollo de los planes y estrategias. 
Involucramiento del empleado. El cliente interno deberá llegar a establecer equipos de trabajo:

$\checkmark$ Desarrollar planes y metas, establecer funciones por áreas, se realizará en la segunda semana de cada mes.
Liderazgo de recursos humanos $y$ marketing. Establecimiento de vínculos entre las áreas por medio de las siguientes acciones:

Comunicados de las asignaciones, impartir las nuevas asignaciones por parte de gerencia cuando sea necesario.

Tabla 2. El alineamiento estratégico

\begin{tabular}{|c|c|c|c|c|c|}
\hline Actividad & $\begin{array}{l}\text { Desarrollo de la } \\
\text { actividad }\end{array}$ & Emplazamiento & Materiales & Costo en Bs.- & Frecuencia \\
\hline $\begin{array}{l}\text { Alineación } \\
\text { con la } \\
\text { estrategia } \\
\text { empresarial. }\end{array}$ & $\begin{array}{l}\text { La empresa elabora y } \\
\text { entrega credenciales } \\
\text { personalizados para cada } \\
\text { trabajador. }\end{array}$ & Empresa & Credenciales & 600 & Única \\
\hline $\begin{array}{l}\text { Identificar } \\
\text { al cliente } \\
\text { interno. }\end{array}$ & $\begin{array}{l}\text { La empresa crea Fichas } \\
\text { individuales con un perfil } \\
\text { básico de gustos y } \\
\text { preferencias e información } \\
\text { del empleado para } \\
\text { fomentar el relacionamiento }\end{array}$ & Empresa & $\begin{array}{l}\text { Folders, } \\
\text { fotografías }\end{array}$ & 60 & Única \\
\hline $\begin{array}{l}\text { Cultura } \\
\text { de la } \\
\text { equidad. }\end{array}$ & $\begin{array}{l}\text { Cada inicio de turno o } \\
\text { jornada, la gerencia y los } \\
\text { clientes internos se } \\
\text { reúnen y repiten juntos la } \\
\text { misión y visión de la } \\
\text { empresa }\end{array}$ & Empresa & $\begin{array}{l}\text { Tarjetas con } \\
\text { la misión y } \\
\text { visión de la } \\
\text { empresa }\end{array}$ & 60 & Unica \\
\hline $\begin{array}{l}\text { Involucrar } \\
\text { a todas } \\
\text { las áreas }\end{array}$ & $\begin{array}{l}\text { Los equipos de } \\
\text { planificación se reúnen } \\
\text { para evaluar el trabajo y } \\
\text { escuchar propuestas en } \\
\text { bien del empoderamiento }\end{array}$ & Empresa & $\begin{array}{l}\text { Material de } \\
\text { escritorio } \\
\text { Refrigerio }\end{array}$ & $\begin{array}{l}100 \\
140\end{array}$ & Mensual \\
\hline
\end{tabular}

Nota: Elaboración propia en base a Regalado, Allpacca, Baca y Gerónimo, (2011) 


\section{Acciones}

Alineación con la estrategia empresarial. La empresa deberá transmitir la importancia de los clientes internos para la misma, de la siguiente manera:

- Elaboración de credenciales personales por cada cliente interno, entrega de las mismas individualmente, explicar el uso de la misma en el trabajo.

Identificar al cliente interno. Elaborar un registro de los clientes internos:

- Elaboración kardex individual, registro de la información solicitada en el kardex como ser; perfiles educativos, socioeconómicos y respuestas personales.

Cultura de la equidad.- Los clientes internos y los empleadores conocerán conjuntamente la misión y la visión de la empresa, para ello se realizara:

- Elaboración de tarjetas individual con la misión y visión de la empresa para cada integrante de la empresa, entrega de los mismos, cada inicio de jornada o turno se reunirán y repetirán la misión y la visión de la empresa.

Involucrar a todas las áreas. Empleadores como clientes internos o el representante designado se reunirá en la planificación.

- Desarrollar metas y planes, el uso de credenciales, memorización de la misión y visión, actualización de kardex, cada cuarta semana del mes.

Tabla 3. La definición de objetivos

\begin{tabular}{|c|c|c|c|c|c|}
\hline Actividad & Desarrollo de la actividad & Emplazamiento & Materiales & Costo en Bs.- & Frecuencia \\
\hline $\begin{array}{l}\text { Identificar } \\
\text { objetivos }\end{array}$ & $\begin{array}{l}\text { Planificación de objetivos } \\
\text { de la empresa sobre las } \\
\text { bases de: funcionalidad, } \\
\text { crecimiento yrentabilidad }\end{array}$ & Empresa & --- & 0 & Mensual \\
\hline $\begin{array}{l}\text { Definir } \\
\text { indicadores }\end{array}$ & $\begin{array}{l}\text { Detallar los indicadores a } \\
\text { ser aplicados. }\end{array}$ & Empresa & --- & 0 & Mensual \\
\hline $\begin{array}{l}\text { Empleado } \\
\text { fidelizado }\end{array}$ & $\begin{array}{l}\text { Verificación por parte de la } \\
\text { gerencia de la recepción y } \\
\text { aceptación de los planes } \\
\text { dirigidos a los clientes } \\
\text { internos. }\end{array}$ & Empresa & --- & 0 & Mensual \\
\hline
\end{tabular}

Nota: Elaboración propia en base a Regalado, Allpacca, Baca y Gerónimo, (2011) 


\section{Acciones}

Identificar objetivos.- Basados en la misión y visión, la gerencia deberá medir los logros y resultados en favor de la empresa.

- Evaluación de la aplicación de la estrategia, basados en; en la inversión de la aplicación de la estrategia, verificación de la percepción del cliente externo, observar y revisar el impacto de esta en la empresa, cada primer semana del mes.

Definir indicadores.- Establecimiento de:

- Metas y planes por cada cliente interno, en puntualidad, diligencia laboral, utilización de los recursos proporcionados por la empresa, cada primera semana del mes.

\section{Empleado fidelizado}

Los objetivos deberán estar diseñados para que el cliente interno no abandone la organización, por ello, se verificara la funcionalidad de los objetivos y su adaptabilidad.

- Evaluación y retroalimentación de la aplicación de las actividades hasta este punto, establecimiento de metas y planes acordes a los requerimientos como ser; uso de sus credenciales y tarjetas, observar y revisar el impacto de esta en la empresa, cada cuarta semana del mes.

Tabla 4. La definición de beneficios

\begin{tabular}{|c|c|c|c|c|c|}
\hline Actividad & Desarrollo de la actividad & Emplazamiento & Materiales & Costo en Bs.- & Frecuencia \\
\hline $\begin{array}{l}\text { Identificación } \\
\text { de beneficios }\end{array}$ & $\begin{array}{l}\text { Beneficios para la empresa } \\
\text { sobre las bases de } \\
\text { Rentabilidad y crecimiento en } \\
\text { base al producto. }\end{array}$ & Empresa & --- & 0 & Mensual \\
\hline $\begin{array}{l}\text { Beneficio } \\
\text { mutuo }\end{array}$ & $\begin{array}{l}\text { Beneficios para los clientes } \\
\text { Internos y la empresa. }\end{array}$ & Empresa & --- & 0 & Mensual \\
\hline \multirow[t]{2}{*}{ Capacitación } & $\begin{array}{l}\text { Se realiza de acuerdo a los } \\
\text { requerimientos que se tenga } \\
\text { dentro la empresa. }\end{array}$ & Empresa & $\begin{array}{l}\text { Facilitador } \\
\text { Certificados } \\
\text { de } \\
\text { participación }\end{array}$ & $\begin{array}{l}250 \\
120\end{array}$ & $\begin{array}{l}\text { Trimestral } \\
\text { Trimestral }\end{array}$ \\
\hline & & & Refrigerio & 140 & Trimestral \\
\hline Integración & $\begin{array}{l}\text { Planificación y } \\
\text { retroalimentación de las } \\
\text { funciones desempeñadas por el } \\
\text { personal en conjunto de toda la } \\
\text { empresa. }\end{array}$ & Empresa & --- & 0 & Mensual \\
\hline $\begin{array}{l}\text { Gestión } \\
\text { humana }\end{array}$ & $\begin{array}{l}\text { El inmediato superior o el } \\
\text { encargado seleccionado de entre } \\
\text { los clientes internos, deberá } \\
\text { representar los requerimientos y } \\
\text { necesidades de sus compañeros. }\end{array}$ & Empresa & --- & 0 & Quincenal \\
\hline
\end{tabular}

Nota: Elaboración propia en base a Regalado, Allpacca, Baca y Gerónimo, (2011) 


\section{Acciones}

Identificación de beneficios. Para ello se realizará lo siguiente:

Establecer metas para la aplicación de la estrategia, con incentivos adicionales por cumplimento de los mismos como ser; bonos, electrodomésticos o artefactos sencillos, descuentos por consumo, víveres, entradas para el cine.

Beneficio mutuo. Relación de reciprocidad de ambas partes.

Se realizará un contrato entre ambas partes, con el objeto de estar vinculados recíprocamente, en el cual estará estipulado la relación contractual de ambos.

Capacitación. El empleador coadyuva en el aprendizaje de sus trabajadores con el fin de incrementar sus capacidades laborales y mejoren su desempeño:

Capacitación en atención al cliente, manipulación de alimentos, inocuidad y limpieza de ambientes, retroalimentación de las anteriores por parte del empleador, entrega de certificados por la capacitación. A realizarse de forma trimestral.

Integración. Verificar y evaluar cómo se está realizando el trabajo en laempresa:

Gerencia y operaciones deberán promover la unión, verificación por medio de la retroalimentación cada cuarta semana del mes.

Gestión humana. El representante de los clientes internos deberá interactuar con el Gerente:

Representación de los clientes internos con el propósito de; mostrar y hacer conocer las necesidades de los clientes internos, con el objetivo de que estas sean atendidas, se realizará cada segunda semana de mes. 
Estrategia de endomarketing para fidelizar a los clientes internos de una empresa de Pizza

Tabla 5. La planeación

\begin{tabular}{|c|c|c|c|c|c|}
\hline Actividad & Desarrollo de la actividad & Emplazamiento & Material es & $\begin{array}{l}\text { Costos } \\
\text { en Bs.- }\end{array}$ & Frecuencia \\
\hline $\begin{array}{l}\text { Comunicación } \\
\text { efectiva }\end{array}$ & $\begin{array}{l}\text { La gerencia y los clientes } \\
\text { internos, mantendrán } \\
\text { una comunicación de ida } \\
\text { y vuelta. }\end{array}$ & Empresa & ---- & 0 & Diaria \\
\hline Segmentación & $\begin{array}{l}\text { Recopilación } \\
\text { información } \\
\text { indispensable para la } \\
\text { base de datos de la } \\
\text { empresa por parte de la } \\
\text { gerencia. }\end{array}$ & Empresa & ---- & 0 & Mensual \\
\hline $\begin{array}{l}\text { Gestión de } \\
\text { resistencias y } \\
\text { grupos de } \\
\text { influencia }\end{array}$ & $\begin{array}{l}\text { Asignación de roles y } \\
\text { tareas relacionadas con } \\
\text { el control de los clientes } \\
\text { internos y la gerencia } \\
\text { para la aplicación de la } \\
\text { estrategia. }\end{array}$ & Empresa & ---- & 0 & Quincenal \\
\hline $\begin{array}{l}\text { Flexibilidad y } \\
\text { adaptabilidad }\end{array}$ & $\begin{array}{l}\text { Tolerancia por parte de } \\
\text { la gerencia con los } \\
\text { clientes internos en la } \\
\text { implementación de la } \\
\text { estrategia. }\end{array}$ & Empresa & ---- & 0 & Diario \\
\hline Evaluación & $\begin{array}{l}\text { Considerar la posible } \\
\text { existencia de variaciones } \\
\text { en la aplicación de la } \\
\text { estrategia para adecuarla } \\
\text { e implementarla. }\end{array}$ & Empresa & --- & 0 & Mensual \\
\hline
\end{tabular}

Nota: Elaboración propia en base a Regalado, Allpacca, Baca y Gerónimo, (2011)

\section{Acciones}

Comunicación efectiva. La estrategia deberá cumplir con:

Se realizará de forma de manera; diaria, semanal, quincenal y mensual, tanto de forma personal como por medio de la representación que tengan los clientes internos.

Segmentación. Delimitar el tipo de clientes internos con los que contamos.

Realizar la modificación, actualización y de los registros de los clientes internos, de forma mensual.

Gestión de resistencias y grupos de influencia.- Formar grupos que jueguen en favor de la empresa:

Rotar asignaciones de representación, de forma quincenal. 
Flexibilidad y adaptabilidad. La estrategia exige cambios en la empresa y clientes internos:

En el conocimiento de la misión y visión, en uso de las credenciales, en la actualización de la información para los kardex. A realizarse de forma diaria.

Evaluación. Cómo está marchando la estrategia en la empresa y realizar los ajustes necesarios si estos fueran requeridos.

Verificar el desarrollo de las distintas actividades, por medio de; representantes asignados de los clientes internos, adaptar, modificar, o actualizar información de los clientes internos, realizar análisis de la aplicación de toda la estrategia de forma mensual, cada fin de mes.

\section{Presupuesto para la implementación}

La implementación de la estrategia precisa de la inversión presupuestaria para poder llegar a solventar las actividades planteadas. Por lo que se considera los aspectos en los cuales se utilizarán de los recursos económicos para dicha implementación tomados de las actividades que anteriormente se describieron que se implementarían.

\section{Beneficios de la implementación}

Uno de los aspectos importantes del presente estudio fue de dar a conocer los beneficios que brinda la implementación de una estrategia de endomarketing para la fidelización de los clientes internos de la empresa pizzería "El Pizarrón" en la ciudad de Potosí, lo cuales se denotan en la tabla 7. 
Tabla 6. Beneficios de la estrategia de endomarketing

\begin{tabular}{cll}
\hline № & Beneficios Identificados & \multicolumn{1}{c}{ Descripción } \\
\hline 1 & $\begin{array}{l}\text { Ambiente laboral cómodo } \\
\text { y distendido }\end{array}$ & $\begin{array}{l}\text { Esto implica prestar atención a los detalles y cuidar el } \\
\text { emplazamiento del trabajador. }\end{array}$
\end{tabular}

2

Fortalece la cultura organizacional de la empresa

3 Mejora la comunicación en la empresa

4 Permite una mejor valoración y evaluación de la productividad del cliente interno

5 Mejora la relación de los clientes internos

6 Unificación de toda la empresa en el alcance de objetivos comunes

7 Fomenta la generación de ideas innovadoras

8 Reducción de errores

9 Aumento de productividad y eficacia
Al realizar las distintas actividades que hacen que los clientes internos conozcan y la misión y visión y se logran identificar con la empresa esto hace que exista una fidelización real. Los clientes internos serán, eternos clientes.

Gracias a la comunicación que es de ida y vuelta se crea una retroalimentación permanente que optimiza los procesos. Haciendo de ello algo vital por marcar en gran medida la eficiencia final de la empresa.

Este aspecto es muy importante, tanto de manera individual como colectiva, dado el mayor interés y observación del cliente interno.

En este punto se puede mencionar el empoderamiento, que define una estrategia basada en aportar más protagonismo y responsabilidad a los clientes internos. De este modo adquieren conocimientos más relevantes, aprenden antes y tienen una actitud más decidida, lo que puede aportar positivamente a la empresa.

Los objetivos al estar diseñados para ser alcanzados, se pueden alcanzar de manera impositiva, pero al estar involucrado de forma directa el cliente interno tanto en el diseño de estos como en la ejecución, estos pueden ser logrados de manera eficaz.

La participación del cliente interno en el desarrollo de la estrategia de endomarketing ocasiona que exista ideas no consideras por la óptica que estos brindan a la gerencia, haciendo que el trabajo en la empresa se más óptimo.

Compartir los proyectos reduce el riesgo de error al ser analizado por una óptica diferente, que en este caso es la del cliente interno.

Al estar involucrado directamente el cliente interno en el desarrollo e implementación de la estrategia de endomarketing, ocasiona que exista un compromiso intrínseco y de pertenecía con la empresa, incrementándose de esta forma el rendimiento de los mismos. 


\begin{tabular}{clc}
\hline № & Beneficios Identificados & Descripción \\
\hline 10 & $\begin{array}{l}\text { Mejora de la atención al } \\
\text { cliente }\end{array}$ & $\begin{array}{l}\text { Teniendo el sentido de pertenencia en la empresa ello mejora el } \\
\text { trato que los clientes internos tienen con los clientes externos. }\end{array}$
\end{tabular}

11 Disminución del retiro de los clientes internos

12 Incremento de la motivación
Al ser íntegramente participes en las decisiones que se toman en la empresa para la misma, existe una alta probabilidad que no se retiren o renuncien, a menos que sean por motivos de fuerza mayor.

El cliente interno al participar de forma directa en la toma de decisiones, al ser escuchada su opinión y esta ser considera a su vez, considerando también los distintos incentivos que le brinda la empresa, ayuda a que sus necesidades puedan estar satisfaciéndose de manera gradual.

Nota: Elaboración propia en base a la planeación de los beneficios de la estrategia de endomarketing

De esta manera se observa que utilizando la estrategia de endomarketing para la fidelización de los clientes internos en la empresa pizzería "El Pizarrón" de la ciudad de Potosí, se obtiene beneficios tanto objetivos como subjetivos en los clientes internos de la empresa.

\section{CONCLUSIONES}

Para finalizar se ha logrado conceptualizar los principios y conceptos de endomarketing y de fidelización del cliente interno, que permitiendo fundamentar teóricamente esta investigación, por lo que se concluye un establecimiento y posicionamiento teórico que permitió orientar el estudio.

Con relación al diagnóstico de la situación actual de la fidelidad de los clientes internos de la empresa pizzería "El Pizarrón", cuya descripción se fundamenta en los datos obtenidos por los instrumentos de recolección de información como la entrevista, la encuesta, la guía de observación, los cuales permitieron determinar que la información recolectada de la fidelización del cliente interno, permite la orientación centrada en una estrategia de endomarketing.

A acuerdo a la estrategia de endomarketing esta permitió fidelizar a los clientes internos, tomándose en cuenta acciones de las cuales sus actividades estuvieron basadas en; involucramiento de la alta dirección, alineamiento estratégico, definición de objetivos, definición de beneficios y la planificación para su posterior implementación, finalizando que la actividades mencionadas son las adecuadas para un posible desarrollo en la empresa.

\section{REFERENCIAS}

Gómez, D. (2014). Qué es endomarketing y sus beneficios. Colombia: bien pensado. Recuperado de: https://bienpensado.com/que-esendomarketing-y-sus-beneficios/

Nayar, V. (2011). Primero los empleados, los clientes después (2a. ed.) Profit editorial. Barcelona; España

Regalado, O., Allpacca, R., Baca, L., y Gerónimo, M. (2011). Endomárketing: estrategias de relación con el cliente interno 